\title{
Sausage mode instability of thin current sheets as a cause of magnetospheric substorms
}

\author{
J. Büchner and J.-P. Kuska \\ Max-Planck-Institute für Aeronomie, D-37191 Katlenburg- Lindau, Germany \\ Received: 14 October 1998 / Revised: 19 January 1999 / Accepted: 22 January 1999
}

\begin{abstract}
Observations have shown that, prior to substorm explosions, thin current sheets are formed in the plasma sheet of the Earth's magnetotail. This provokes the question, to what extent current-sheet thinning and substorm onsets are physically, maybe even causally, related. To answer this question, one has to understand the plasma stability of thin current sheets. Kinetic effects must be taken into account since particle scales are reached in the course of tail current-sheet thinning. We present the results of theoretical investigations of the stability of thin current sheets and about the most unstable mode of their decay. Our conclusions are based upon a non-local linear dispersion analysis of a crossmagnetic field instability of Harris-type current sheets. We found that a sausage-mode bulk current instability starts after a sheet has thinned down to the ion inertial length. We also present the results of three-dimensional electromagnetic PIC-code simulations carried out for mass ratios up to $M_{i} / m_{e}=64$. They verify the linearly predicted properties of the sausage mode decay of thin current sheets in the parameter range of interest.
\end{abstract}

Key words. Magnetospheric physics (plasma waves and instabilities; storms and substorms) · Space plasma physics (magnetic reconnection)

\section{Introduction}

Magnetospheric substorms correspond to a configurational instability of the magnetotail (e.g. Baker et al., 1998) which is closely related with reconnection (Russell and McPherron, 1973; Hones, 1979; Baker et al., 1996;

Correspondence to: J. Büchner, e-mail: buechner@linmpi.mpg.de
Vasyliunas, 1975; Axford, 1984). The question is, what triggers reconnection in the collisionless plasma of the Earth magnetotail and whether it is the cause or the consequence of substorm onsets. In the past, a spontaneous tearing mode instability of the tail current sheet was considered to be a cause of substorms and reconnection (Coppi et al., 1996). However, the collisionless tearing mode alone, even ion tearing (Schindler, 1974) or the electron-chaos driven instability (Büchner and Zelenyi, 1987) is stable in two-dimensional tail-like configurations (Pellat et al., 1991; Pritchett, 1994; Pritchett and Büchner, 1995). The restrictions do not apply, if one turns to the more realistic consideration of all three spatial dimensions (Büchner, 1995). In addition, it is known from observations that thin current sheets are formed in the near-Earth magnetotail just prior to substorm onsets (Kaufmann 1987; Sergeev et al., 1990; Mitchell, 1990; Pulkkinen et al., 1992; Schindler and Birn, 1993; Sanny et al., 1994). In the past the evolution of thinning current sheets has been described in the MHD approach (see Wiegelmann and Schindler, 1995). The question has remained open, at which threshold thin current sheets become unstable and what is the mode of their decay? Since the observed sheet thicknesses prior to substorm onset reach the characteristic scales of the particle, at least of the ion motion, a kinetic approach is necessary to describe the sheet stability and its transition to instability. Both, from the current stability and three-dimensionality of reconnection points of view the current direction should be a special focus of future investigations.

In the past mainly local approaches to current stability were used. Motivated by the search for possible mechanisms of "anomalous resistivity", current instabilities were thought to reduce the free energy of the current flow. This could be, for example, the ionacoustic instability (Coroniti and Eviatar, 1977). Unfortunately, such electrostatic waves are damped away in the magnetotail plasma where $T_{i}>T_{e}$. Also, they would be pretty much localized near the center of the sheet. Their relevance for reconnection was, therefore, 
disregarded, as well as the concept of a resulting anomalous resistivity (e.g., Coroniti, 1985). Alternatively, gradient driven instabilities were considered (e.g., Huba et al., 1977). These would be due to the strong density gradient at the edge of the current sheet which provides another possible source of free energy in current sheets. The resulting lower hybrid drift instability is, however, damped away in the high- $\beta$, weak magnetic field region in the center of the sheet, the critical diffusion region of reconnection (Huba et al., 1980). Further, electromagnetic cross-field instabilities were looked for (Kuznetsova and Nikutowski, 1994; Lui et al., 1991). While all these investigations were based on local instability analyses, plasma simulations automatically take into account also non-local effects of the current sheet decay. In their simulations Winske (1981a, b), nevertheless, saw just the lower-hybrid drift instability at the edges of the sheet. Brackbill et al. (1984) simulated the sheet evolution over a somewhat longer time. They observed that after the lower-hybrid drift wave, an unstable driftkink mode evolves. They interpreted it as a non-linear stage of the lower-hybrid drift instability. More advanced multi-dimensional particle code simulations found later that in thin current sheets unstable bulk eigenmodes of the system can also evolve on their own. This way both an asymetric-kink (Zhu and Winglee, 1996; Ozaki et al., 1996; Pritchett et al., 1996; Lapenta and Brackbill, 1997) and a symmetric-sausage mode (Büchner and Kuska, 1996, 1997) were found. It became obvious that whole, thin-current sheets decay in an essentially non-local bulk mode, detailed properties of which remained, however, unclear.

Non-local analytical investigation of the linear stability properties of thin currents sheets were carried out by Pritchett and Coroniti (1996) in a two-fluid approach. They did not find the eigenfunctions of the mode but solved the fluid equations as an initial value problem. This approach takes into account such nonMHD effects as the electron-ion cross-field streaming and finite electron inertia. It is limited, however, to longer wavelengths with wave numbers $L_{z} k \ll 1$ (normalized to $L_{z}$, the sheet's half-width). For unequal ion and electron masses the initial value solution of the twofluid equations revealed a complex eigenfrequency $\omega=\omega_{r}+i \gamma$. The growth rate of the instability $\gamma$ increases with increasing mass ratio from $\gamma=0.25 \Omega_{0, i}$ for an artificial mass ratio $M=M_{i} / m_{e}=4$ to $\gamma \rightarrow \Omega_{0, i}$ for $M \approx 100$. The wave number of the fastest growing mode was $L_{z} k \approx 2 / \sqrt{M}$ and the real frequency varied between $\omega_{r} \approx 0.4 \Omega_{0, i}$ for $M=4$ and $35 \Omega_{0, i}$ for $M=100$. This corresponds to a propagation speed $\omega_{r} / k=0.16 \ldots 0.7 \Omega_{0, i} L_{z}$ for mass ratios between $M=4$ and $M=100$. Yoon et al. (1998) derived and solved the two-fluid equations as an eigenvalue problem. Their solution does not reconcile the results of Pritchett and Coroniti (1996). It is, on the other hand, also restricted to the short wavelength limit. The reason is that resonance effects are not taken into account in fluid approaches, However, particle resonance effects may cause growth and damping of plasma oscillations in collisionless plasmas. Hence, one has to consider the cross-field instability kinetically, taking into account the specifics of the particle motion in current sheets. A first attempt to describe the consequences of the nongyrotropic meandering particle motion in current sheets. A first attempt to describe the consequences of the nongyrotropic meandering particle motion in current sheet magnetic fields (Speiser, 1965) in the framework of a non-local current sheet instability theory was undertaken by Yamanaka (1978). They considered a compressional plasma wave propagating perpendicular to the magnetic field. Another attempt was reported by Lapenta and Brackbill (1997). Although they did not derive from the wave equations a dispersion relation as an eigenvalue problem they obtained a symmetric sausage-mode of the most unstable wave. In contrast to their theoretical discussion, their simulations, however, resulted in an antisymmetric (kink-) mode instability.

We have now analytically derived a linear kinetic dispersion relation of the cross-magnetic field instability from the wave equations of a non-local mode propagating in thin Harris-equilibrium current sheets. Here in this paper we apply those results to mass ratios $M=M_{i} / m_{e}$ up to 64 to compare them directly with high-resolution fully kinetic, electromagnetic kinetic PIC-code plasma simulations. First, in sect. 2, we describe our model and the results of the theoretical analysis of the stability of thin current sheets. In sect. 3 we present the setup of our kinetic PIC code simulations the results of which we discuss in sect. 4. Finally, in sect. 5 we draw conclusions for the onset of magnetospheric substorms.

\section{Theoretical results}

For collisionless plasmas, where particle interactions are permitted mainly by their collective electromagnetic fields instead of binary collisions, the Vlasov equation provides an appropriate tool for analyzing plasma stability:

$$
\left\{\frac{\partial}{\partial t}+(\mathbf{v} \cdot \nabla)+\frac{e_{j}}{m_{j}} \cdot\left(\mathbf{E}+\frac{\mathbf{v} \times \mathbf{B}}{c}\right) \nabla_{\mathbf{v}}\right\} f(t, \mathbf{r}, \mathbf{v})=0
$$

A plasma systems' stability is investigated by linearizing Eq. (1) with respect to small perturbations of an appropriate initial state. For such analysis, it is appropriate to integrate the action of a field perturbation along the unperturbed particle trajectories which are characteristics of the Vlasov Eq. (1). Particle trajectories in current sheet magnetic field reversals are, however, quite complicated (cf., Speiser, 1965). This applies more to thin current sheets, where the ion Larmor radii are comparable with the sheet thickness. In such configurations, most of the free energy is stored and currents are carried by particles which meander across the sheet midplane. The motion on these orbits is nongyrotropic, it can be quasi-adiabatic and even chaotic (Sonnerup, 1971; Büchner and Zelenyi, 1989). The velocity distribution of these non-gyrotropically meandering particles is well described by a drift Maxwellian. The Harris 
(1962) model describes an appropriate thin current sheet equilibrium:

$$
\begin{array}{r}
f_{o j}(\mathbf{r}, \mathbf{v})(\mathbf{r}, \mathbf{v})=n_{o j} \cdot \frac{m_{j}}{2 \pi k_{B} T_{j}} \cdot \exp \frac{W_{j}-u_{d j} P_{j}}{k_{B} T_{j}} \\
=\frac{n_{j}(Z)}{(2 \pi)^{3 / 2} \cdot v_{t j}^{3}} \cdot \exp \left\{-\frac{v_{x}^{2}+\left(v_{y}-u_{j}\right)^{2}+v_{z}^{2}}{2 v_{t j}^{2}}\right\}
\end{array}
$$

where

$n_{j}(Z)=n_{e}(Z)=n_{i}(Z)=n_{o} \cdot \cosh ^{-2}\left(\frac{Z}{L_{z}}\right)$

and

$L_{z}=\frac{\sqrt{k_{B} \cdot\left(T_{e}+T_{i}\right)}}{\sqrt{2 \pi n_{o} e^{2}}} \cdot \frac{c}{u_{d i}-u_{d e}}$

Notice that here in our paper $v_{t j}$ is determined as $v_{t j}=\sqrt{\frac{k_{B} T_{j}}{m_{j}}}$. Further, $e$ is the elementary charge, $m_{j}$ are particle masses and $u_{d j}$ drift velocities in the current direction. The indices $j=e, i$ denote electrons and ions, respectively. For example $T_{e}$ and $T_{i}$ are the electron and ion temperatures. $c$ the speed of light, $k_{B}$ the Boltzmann constant. The self-consistent magnetic field of a Harrisequilibrium

$\mathbf{B}=B_{o} \cdot \tanh \left(\frac{Z}{L_{z}}\right) \cdot \mathbf{e}_{x}$

balances the plasma pressure of the sheet, i.e.

$B_{o}=\sqrt{8 \pi \cdot n_{o} \cdot k_{B} \cdot\left(T_{e}+T_{i}\right)}$.

The thermal Larmor radius in this field is $\rho_{o j}=v_{t j} / \Omega_{o j} \cdot \Omega_{o j}=e B_{o} / m_{j} c$ is the gyro-frequency in the asymptotic field. If the electron and ion drift velocities $u_{d e}$ and $u_{d i}$ are related as $u_{d e} / u_{d i}=-T_{e} / T_{i}$ the electric field vanishes in a Harris equilibrium. In the coordinate system, where this relation of the drift speeds is fulfilled, the half-width of the Harris equilibrium can be rewritten as

$$
\begin{aligned}
L_{z} & \cdot \sqrt{1+\frac{T_{e}}{T_{i}}}=\frac{\sqrt{k_{B} \cdot T_{i}}}{\sqrt{2 \pi n_{o} e^{2}}} \cdot \frac{c}{u_{d i}} \\
& =\sqrt{2} \cdot \frac{v_{t i}}{u_{d i}} \cdot \frac{c}{\omega_{p i}}=\sqrt{2} \cdot \frac{v_{t i}}{u_{d i}} \cdot \lambda_{i i}=\sqrt{2} \cdot \frac{c}{u_{d i}} \cdot \lambda_{D i}
\end{aligned}
$$

where $\lambda_{i i}=c / \omega_{p i}$ is the ion inertial length and $\lambda_{D i}=\sqrt{k_{B} T_{i} / 4 \pi n_{o} e^{2}}$ the Debye length based on the maximum density $n_{o}$ and the ion temperature. Due to the pressure balance Eq. (5) the sheet thickness is, independent on the temperature ratio $T_{e} / T_{i}$, directly related to the thermal ion Larmor radius $\rho_{0, i}$ as

$L_{z}=2 \cdot \rho_{0, i} \cdot \frac{v_{t i}}{u_{d i}}$

We have investigated the stability of the current sheet by solving the Vlasov Eq. (1) for small $\left(\delta f_{1 j} \ll f_{o j}\right)$ perturbations $\delta f_{1 j}$ of the equilibrium distribution function $f_{o j}(\mathbf{r}, \mathbf{v})$ in response to appropriate field perturba- tions, given in terms of small variations $\delta \mathbf{A}$ and $\delta \varphi$ of the electromagnetic potentials. Since we look for a resonant current instability we assume a propagation parallel to the current flow with finite wave numbers $k=k_{y}$. To find the most unstable modes it is appropriate to analyze the response of the Fourier-components of the perturbed distribution function

$\delta f_{1 j}=f_{1 j} \cdot \exp \{-i \omega t+i k Y\}$

The most probable polarization of the wave is characterized by a perturbation of the $Y$-component of the vector potential (Yamanaka, 1978)

$\delta A_{y}=A_{1}(Z) \exp \{-i \omega t+i k Y\}$

while a polarization with non-vanishing $\delta A_{x}$ is most unlikely to grow. Since we are looking for waves propagating slower than the electron thermal velocity, we can neglect the perturbation of the electrostatic potential, i.e. $\delta \varphi \rightarrow 0$. The perturbation $\delta A_{z}$ immediately follows from the Lorenz gauge condition $\nabla \delta \mathbf{A}=0$. With the particle trajectories being characteristics of the Vlasov Eq. (1), the perturbation of the distribution function $f_{o j}(\mathbf{r}, \mathbf{v})$ is obtained by integrating the influence of the field perturbations along the unperturbed particle orbits. In general, there are two kinds of qualitatively different orbits in Harris sheets, non-crossing the neutral plane gyrations and those meandering across the neutral plane (Speiser, 1965). The most important resonances are due to interactions with the particles drifting on meanderorbits. As a result the perturbation, the distribution function $\delta f_{1 j}$ consists of a reversible part $\delta f_{1 j}^{\text {rev }}$ which is due to non-resonant interactions with the inductive electric field and of an irreversible part $\delta f_{1 j}^{\text {irr }}$ which is due to resonant interactions with the meandering particles. From the reversible perturbation $\delta f_{1 j}^{r e v}$ one obtains the corresponding current perturbation

$\delta j_{1}^{r e v}=\Sigma_{j} \frac{e_{j}^{2}}{c k_{B} T_{j}} \int_{\mathbf{v}} \mathrm{d} \mathbf{v} v_{y} \delta A_{y} u_{d j}$,

while the resonant variation of the distribution function $\delta f_{1 j}^{i r r}$ reveals the irreversible current perturbation

$$
\begin{aligned}
\delta j_{1 j}^{i r r}= & \frac{4 \pi}{c} \delta f_{1 j}^{i r r}(Z)=\frac{4 \pi e_{j} \cdot f_{0 j}(Z)}{c^{2} c \cdot k_{B} T_{j}} \\
& \times \int_{-\infty}^{t} \mathrm{~d} t^{\prime}\left[v_{y} \cdot \frac{\partial \delta A_{y}}{\partial t^{\prime}}+u_{d j} v_{y} \frac{\partial \delta A_{y}}{\partial Y}\right]
\end{aligned}
$$

As a result the Vlasov equation leads to a wave equation for the Fourier component $A_{1}(Z)$ of the perturbed vector potential

$$
\begin{aligned}
{\left[\frac{\mathrm{d}^{2}}{\mathrm{~d} Z^{2}}\right.} & \left.+\frac{\omega^{2}}{c^{2}}-k^{2}+\frac{2}{L_{z}^{2} \cosh ^{2}\left(Z / L_{z}\right)}\right] A_{1}(Z) \\
& =-\frac{4 \pi A_{1}(Z)}{c k_{B}} \sum_{j} \frac{e_{j}^{2}}{T_{j}} \int_{\mathbf{v}} \mathrm{d} \mathbf{v} f_{o j} v_{y}^{2} \frac{\omega-k u_{d} j}{k v_{y}-\omega}
\end{aligned}
$$


Equation (12) is a nonlinear integro-differential equation. In order to find the mode structure one can solve it is an eigenvalue problem with respect to $\omega^{2}$ for which the eigenfunctions $A_{1}(Z)$ have to be found. Notice that the operator in the left hand side (1.h.s) of Eq. (12) differs essentially from the corresponding operator in the differential equation of the collisionless tearing mode problem. It contains a finite real frequency $\omega_{r} \neq 0$ and a wave number $k=k_{y}$ instead of $k_{x}$ responsible for sheet tearing. The right hand side (r.h.s.) of Eq. (12) represents the irreversible current perturbations which are due to wave-particle resonances described by Eq. (11). For the further treatment of Eq. (12) we expanded its r.h.s. to a power series with respect to $\omega$ around $\omega=0$. Terms higher then quadratic in $\omega$ were neglected. This approach revealed the following linear wave equation (in dimensionless variables $\zeta=Z / L_{z}$, $v=L_{z} \omega / c, \kappa=L_{z} k$ where $k=k_{y}, \omega_{j}=u_{d j} / c, \sigma_{j}=v_{t j} / c$, $\left.\mu=e / M_{i}, \chi=\frac{L_{z}^{2} n_{o} e_{j}^{2}}{c M_{i}}\right)$

$$
\begin{aligned}
& A_{1}^{\prime \prime}(\zeta)+\left[v^{2}\left(1+\phi_{0} \cosh ^{-2}(\zeta)\right)\right. \\
& \left.\quad-\kappa^{2}+\phi_{2} \cosh ^{-2}(\zeta)\right] A_{1}(\zeta)=0
\end{aligned}
$$

with $u=u_{\mathrm{i}}, \phi_{0}=2+\frac{8 \pi u^{2} \chi}{\sigma_{\mathrm{i}^{2}}}$ and

$\phi_{2}=-\frac{8 \pi \chi}{\kappa^{2} \sigma_{\mathrm{i}}^{2}}+\frac{2 \sqrt{2} \pi^{\frac{3}{2}} u \chi}{\kappa^{2} \sigma_{\mathrm{i}}^{3}}\left[\frac{-i+\operatorname{erfi}\left(\frac{u}{\sqrt{2} \sigma_{\mathrm{i}}}\right)}{e^{\left(u^{2} / 2 \sigma_{\mathrm{i}}^{2}\right)}}+\frac{\sqrt{\mu}\left(i+\operatorname{erfi}\left(\frac{u \sqrt{\mu}}{\sqrt{2} \sigma_{\mathrm{i}}}\right)\right)}{e^{\left(u^{2} \mu / 2 \sigma_{\mathrm{i}}^{2}\right)}}\right]$

From Eq. (13) we have derived the linear dispersion relation by analytically solving the differential equations for the eigenvalues $v_{n}$ with their corresponding eigenvectors $A_{1}^{(n)(Z)}$. In order to solve the generalized eigenvalue problem algebraically and to avoid the slab approximation applicable only in the thick-sheet limit, we applied a system of base functions which smoothly cover the whole spatial domain of interest. In order to compare with kinetic simulation results, let us here consider solutions of this linear dispersion relation for the first six eigenvalues in for mass ratios ranging from $M=\mu^{-1}=M_{i} / m_{e}=1$ to $M=64$ and for $T_{e}=T_{i}$. For a mass ratio $\mu=1 / 64$ the maximum imaginary part $\max _{n}(\operatorname{Imv})$, i.e., the maximum growth rate, is shown in contour plot Fig. 1 as a function of the normalized wave number $L_{z} k$ and of the inverse sheet thickness $1 / L_{z}$, normalized to the ion Larmor radius in the external field $\rho_{0, i}$. The spacing of the contour lines in Fig. 1 can be directly read from Fig. 2, where the (normalized) values of the growth rate are explicitly given for $\rho_{0, i} / L_{z}=1.05$. Figure 2 shows that darker regions in Fig. 1 correspond to larger growth rates. White regions correspond to negative $\gamma$, i.e., to damping instead of growth. The black island to the left of Fig. 1 corresponds to a resonance with the lighter particles (the "electrons"). The "electrons" resonance can be recognized only for artificially small mass ratios much smaller than the real one. They disappear for higher, more realistic mass ratios. The black island to the right of the plot corresponds to ion resonances. They reveal large positive $\gamma \propto \operatorname{Im} v$ which remain even for the much higher realistic mass ratio (cf. Fig. 2). The plot shows that the sheet becomes unstable

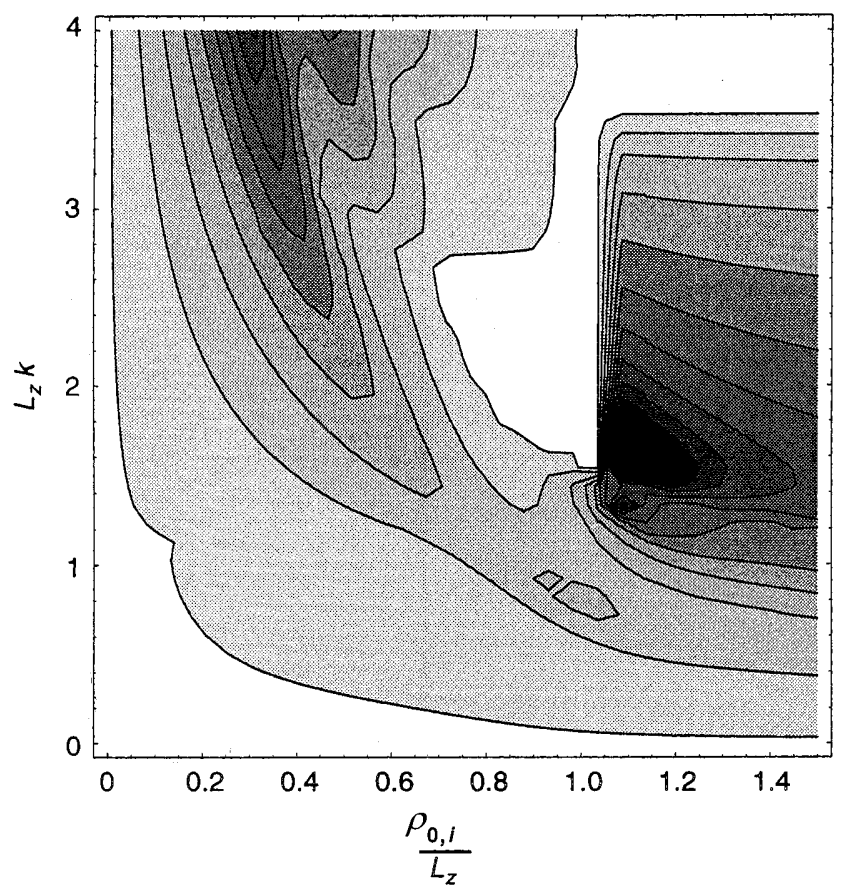

Fig. 1. Contour plot of maximum growth rates $\max _{n}\left(\operatorname{Im} v_{n}\right)$ as a function of wave number $L_{z} k$ and inverse sheet thickness, normalized to the ion gyroradius $\rho_{0, i}$ (for a mass ratio $\mu=1 / 64$ )

(positive $\gamma \propto \operatorname{Imv}$ ) due to ion resonances after it has thinned down to $L_{z} \approx \rho_{0, i}$.

Figure 3 depicts isocontours of the maximum growth rate $\max _{n}\left(\operatorname{Imv}_{n}\right)$ for marginally thin sheets $\left(L_{z} / \rho_{0, i}=1\right)$ as a function of the mass ratio $\mu$ (and of the normalized wave number $L_{z} k$ ). The figure shows that the damping

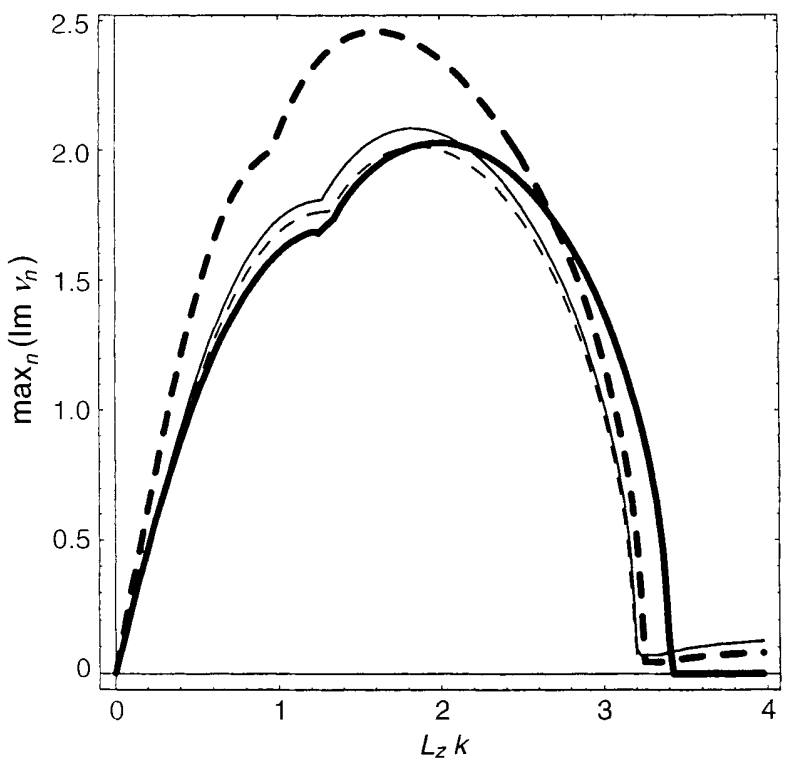

Fig. 2. The growth rate $\max _{n}\left(\operatorname{Im} v_{n}\right)$ for marginally thin sheets $\left(\rho_{o, j} / L_{z}=1.05\right)$ as a function of the wave number, depicted by a thick solid line, a thick dashed line, a thin solid line and a thin dashed line for four different mass ratios $M=\mu^{-1}=1,4,16$, and 64, respectively 


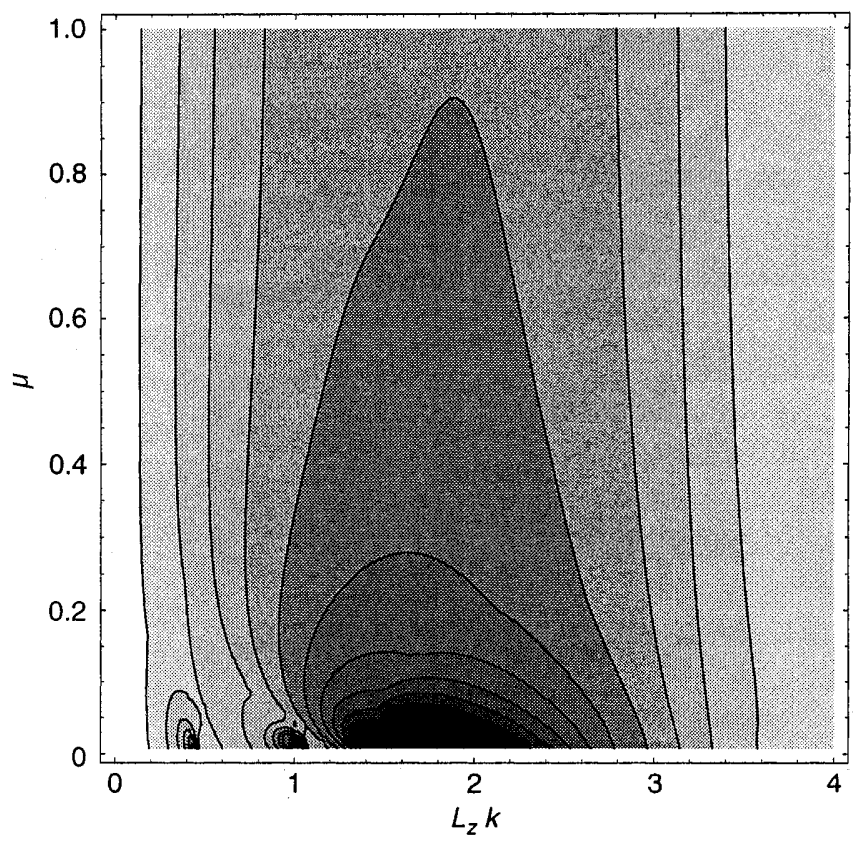

Fig. 3. Contour plot of maximum growth rates $\max _{n}\left(\operatorname{Im} v_{n}\right)$ as a function of wave number $L_{z} k$ and mass ratio $\mu$ for a marginally thin sheet $\left(\rho_{0, i}=1.05 L_{z}\right)$

influence of the negatively charged, lighter particles "electrons") on the wave growths is restricted to artificially large relative mass ratios $m_{e} / M_{i}>0.1$. For $\mu<0.1$ the fastest modes and in the wave number range $L_{z} k=1.2 \ldots 2$ already grow at the high rates, shown in Fig. 2. This figure depicts the growth rate $\max _{n}\left(\operatorname{Im} v_{n}\right) \cdot L_{z} / c$ of the fastest growing modes for the four different mass ratios $M=\mu^{-1}=1,4,16$ and 64 as a function of the wave number $L_{z} k$ for a marginally thin sheet $\rho_{0, i}=1.05 L_{z}$. This verifies the different influence of electrons and ions on the instability. It confirms that for realistic mass ratios the electron damping can be neglected while the ion influence dominates the wave growth. The wave number of the most unstable mode shifts towards shorter wavelength, from $L_{z} k=1.0$ towards 2. The propagation speed $\omega_{r} / k$ of the fastest growing mode approaches the ion drift speed which allows highly efficient ion resonances. From the symmetry of the eigenfunctions $A_{1}^{(n)}(Z)$ which correspond to the fastest growing wave follows its symmetric about the current sheet. This reminds the structure of the ideal MHD sausage mode instability. Hence we called it a kinetic sausage mode.

\section{Plasma simulations}

We have run our fully kinetic electromagnetic PIC-code (Birdsall and Langdon, 1991) GISMO in extended parameter regimes (cf. Büchner and Kuska, 1996) to verify whether the linear dispersion analysis provides correct hints at the fastest growing instability of thin current sheets. GISMO integrates the classical equations of relativistic particle motion selfconsistently with the wave equations for the scalar and vector potentials $\phi$ and
A of the electromagnetic field. This has several advantages. The most notable one is the easy implementation of an implicit integration scheme and the simple formulation of the boundary conditions. The field solver is implicit and unconditionally stable for all step sizes. The boundary conditions for the potentials at the edges of the simulation box $0 \leq x \leq \ell_{x}, 0 \leq y \leq \ell_{y}, 0 \leq z \leq \ell_{z}$ are given by

$w(x, y, z)=w\left(x+\ell_{x}, y, z\right)$,

$w(x, y, z)=w\left(x, y+\ell_{y}, z\right)$,

$w(x, y, 0)=w\left(x, y, \ell_{z}\right)=0$.

These are periodic boundary conditions in the $x$ and $y$ directions. The potentials are zero at the upper and lower boundaries. The Laplacian of the field solver is approximated by a finite difference scheme. This yields a sparse linear system of equations which is solved at every time step by successive overrelaxation with a conjugate gradient preconditioner. The iterative solution starts with the potential of the previous time step. The initial guess for $w^{(n+1)}$ is usually very close to the solution, so only a few iterations are needed to obtain the desired accuracy. Electric and magnetic fields are calculated from the potentials by finite difference expressions for the derivatives. The continuous electric and magnetic fields are obtained by a trilinear interpolation of the data between the mesh points. Since the particle motion across the boundaries of the simulation box violates the gauge condition

$$
\frac{1}{c} \frac{\partial \phi}{\partial t}-\nabla \mathbf{A}=0
$$

the forces are corrected as soon as the error in the gauge exceeds the error in the iterative solution of the field solver. Charge density $\varrho$ and current density $\mathbf{j}$ are calculated from the positions and velocities of the particles. The particle orbits are calculated by solving the relativistic equations of motion. With the maximum simulation box dimension $\ell=\max \left\{\ell_{x}, \ell_{y}, \ell_{z}\right\}, \mathbf{v}^{*}=\mathbf{v} / c$ and $\tau=\ell t / c$. The solution of the equations of motion is obtained using an explicit embedded Runge-Kutta pair of order 4(3) with step size control (Deuflhard and Bornemann, 1994). This is the classical Runge-Kutta method but with an embedded third order step. The fourth order integration scheme ensures that the particle gyration in the magnetic field is resolved properly. GISMO controls the step size in the particle integrator. In fact, the accuracy of the integration is an often overlooked quantity in kinetic simulations. However, the correct modeling of a dynamic system requires a stable and accurate solution, the accuracy is even the more important quantity. The step size control by GISMO ensures both that the solution keeps stable and that the local truncation error

$[\hat{\epsilon}]=\left(\xi-\xi^{*}\right) / 6$

is lower than the desired accuracy for all trajectories (here $\xi$ and $\xi^{*}$ are the fourth and third order approximations obtained by the embedded Runge-Kutta pair). For the modelling of an instability the step size control speeds up 
the calculation during the initial metastable phase of the system evolution. Here the classical Runge-Kutta method has the advantage that it can be implemented in a memory efficient way. In addition to memory saving, the classical Runge-Kutta method contains two redundant steps in the middle of the integration interval and at the end. This improves the convergence of the solution for the particle motion. Higher order Runge-Kutta integrators (Cash and Karp, 1990; Hairer et al., 1987; Verner, 1993) would not improve the integration quality further since the force term is calculated by a lower order interpolation. The maximum local error of the integration was chosen to be $\hat{\epsilon}<1 \cdot 10^{-5}$. Since the motion of the particles near the boundary must be consistent with the fields periodic particle boundary conditions are assumed in the $x$ - and $y$ directions. The few particles which reach the boundaries in the $z$ direction are randomly replaced inside the box in accordance with the original distribution function.

\section{Simulation results}

We initialized a Harris-type equilibrium distribution described by Eqs. (2)-(4) to verify the properties of the unstable decay of thin current sheets. We extended the size of the simulation box up to $12 L_{z} \times 12 L_{z} \times 6 L_{z}$ gridplanes (where $L_{z}$ is the half-thickness of the sheet, cf. Eq. (4)). In comparison with our previous simulation runs (e.g. Büchner and Kuska, 1996, 1987) we enhanced the grid resolution of the Debye length up to a factor of three. The electromagnetic fields are calculated on a mesh of $128 \times 128 \times 64$ gridplanes in the $X, Y$ and $Z$ directions, respectively, and the number of particles was increased up to $6 \cdot 10^{6}$. The main result of our higher resolution simulations is that, as in our previous runs at lower resolution with fewer particles and in smaller simulation domains (Büchner and Kuska, 1996, 1987), a sausage mode instability evolves, which is symmetric about the current sheet. None of our simulations without additional background plasmas showed a faster growing of a kink mode. As an example, we present here in this paper results obtained by simulating critically thin sheets $\left(L_{z}=\rho_{0, i}\right)$ with equal initial electron and ion temperatures $\left(T_{e}=T_{i}\right)$, without additional plasma background for both a mass ratio of $M=M_{i} / m_{e}=\mu^{-1}=1$ and $M=\mu^{-1}=64$. For three moments of time Figs. 4 and 5 depict snapshots of isodensity (density level: $2 / 3$ of the maximum) surfaces of the ions in an the evolving bulk current sheet instability. Figure 4 corresponds to a mass ratio $1: 1$ and Fig. 5 to $1: 64$. The box sizes are given in terms of $\Delta=12 L_{z}$. Our linear dispersion investigations had shown that for a mass ratio $M=1$ the main contribution to the structure formation corresponds to the lowest order eigenvalue $v_{0}$. The real part of $v_{0}$ vanishes in the range of $0.9 \leq L_{z} k \leq 1.16$. Hence the last allowed wave vector $k$ value corresponds to a wave number $L_{z} k \approx 0.9$. Assuming that the most important contribution corresponds to the eigenvalue with the maximum imaginary part, the eigenvalues of Eq. (13) suggest a wave length of the fastest growing mode $\lambda \approx 2.2 \pi L_{z}$. Since the box length in the current
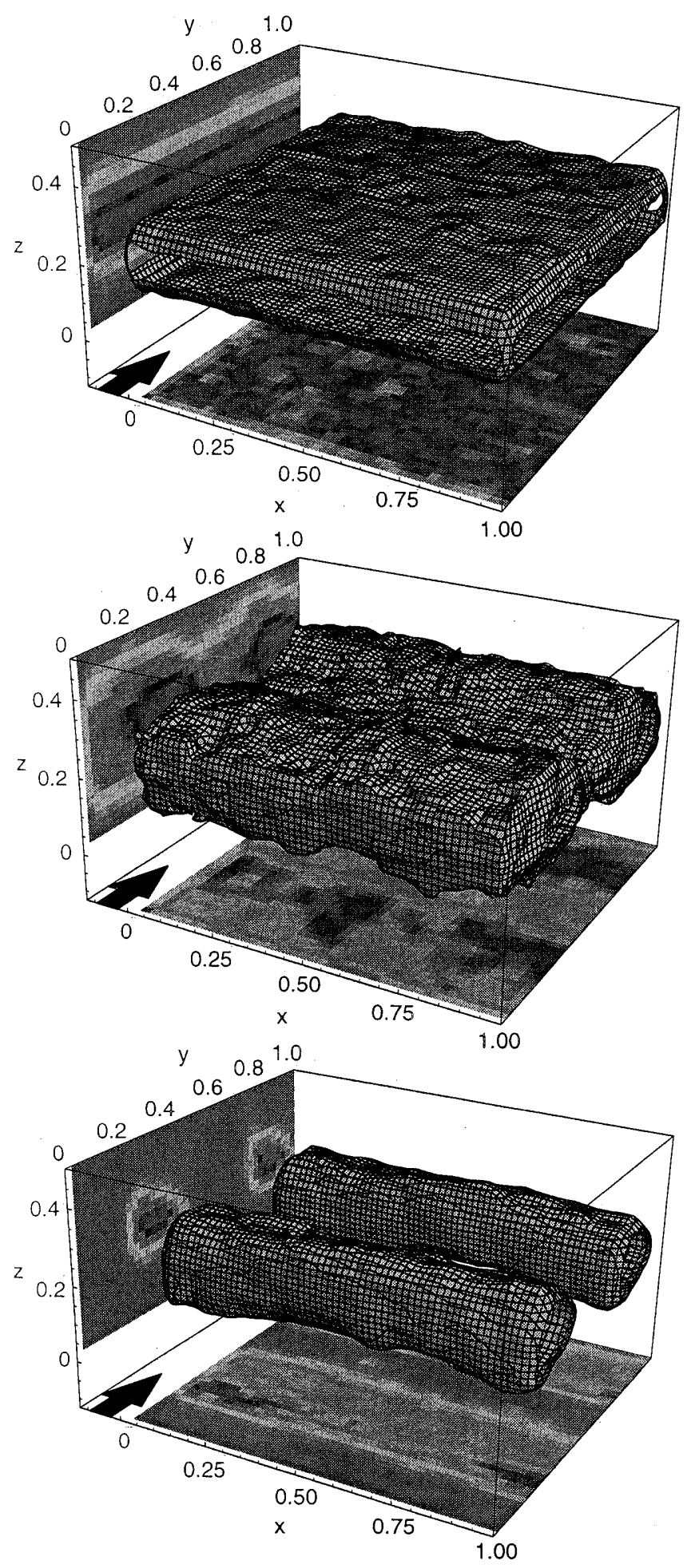

Fig. 4. Instability of a three-dimensional Harris sheet equilibrium for a mass ratio $M=1$. The images show ion density isosurfaces at $t \Omega_{0}=1.4,9.8$ and 19.6, respectively. The left box side shows isodensity contours for a cut through the box at $x=6 L_{z}=0.5 \Delta$. The bottom plane shows isodensity contours for a cut through the box at $z=3 L_{z}=0.25 \Delta$

direction $\Delta=1$ corresponds to $12 L_{z}$ it should accommodate about two wavelength. From Fig. 4 one sees that the theoretically predicted wavelength agrees well 

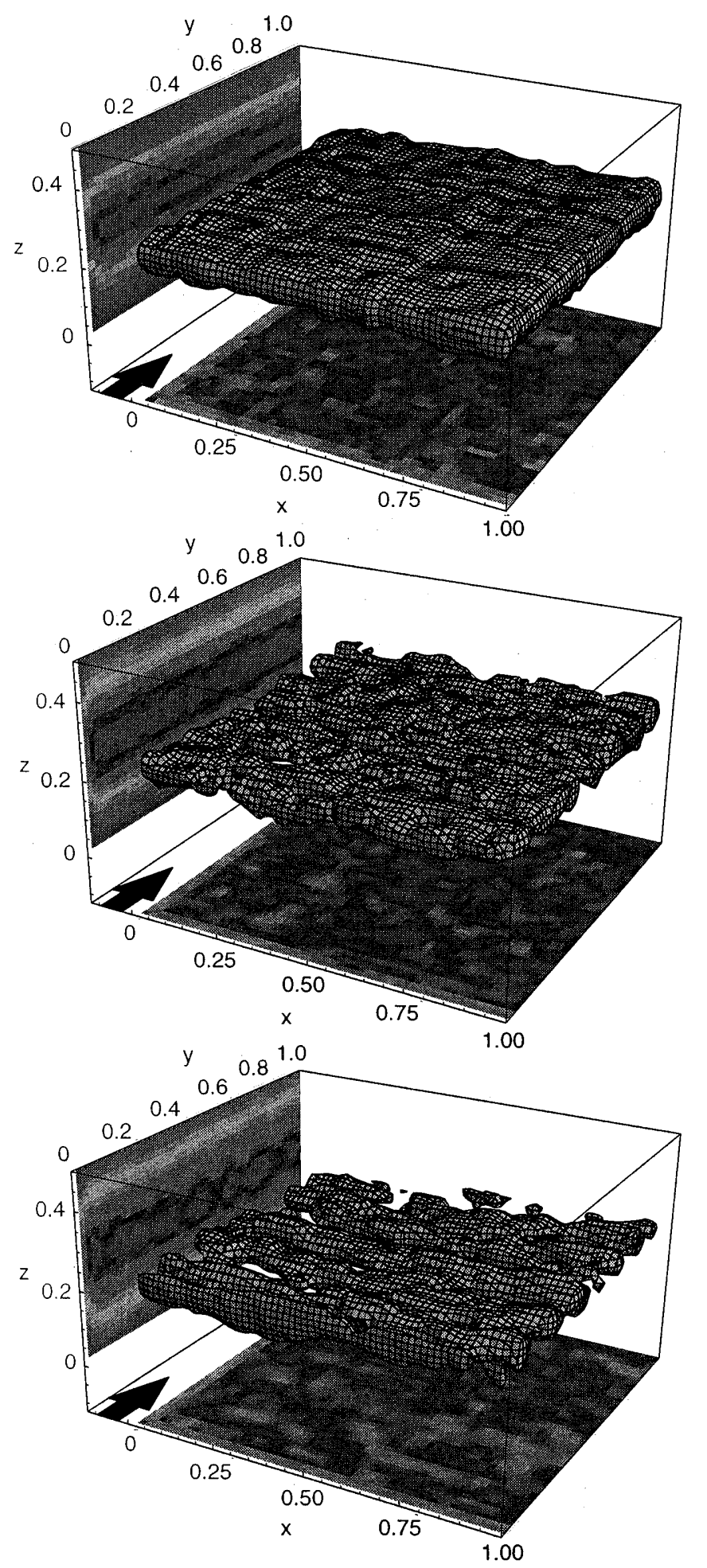

Fig. 5. Same as Fig. 4, but for a mass ratio $M=64$

with the result of the numerical modeling. For mass ratios $M \gg 1$ the positive real parts of the eigenvalues $v_{n}$ are not restricted to $L_{z} k$ values less than one and shorter wavelength modes can grow. For $M \gg 1$ the maximum imaginary part of the eigenvalues of the linear dispersion relation corresponds to a wave number $L_{z} k \approx 2$, this is a wavelength $\lambda \approx 1.05 \pi L_{z}$. Figure 5 shows that for a mass ratio $M=64$ the bulk mode wavelength of the spatial structure is, indeed, close to $4 \lambda$ fitting in a $12 L_{z}$ long box. This agrees well with the linear dispersion result $L_{z} k \approx 2$. The same can be said about the propagation speed and the wave frequency: the wave propagates, as theoretically predicted, with the ion drift speed and the wave frequency gets close to the ion cyclotron frequency. The growth rate of the instability is of the order of the ion cyclotron frequency, too. This makes the sausage mode instability grow much faster than two-dimensional collisionless tearing mode instability of thin current sheets.

\section{Summary, discussion and conclusions}

We reported results of analytical and numerical kinetic investigations of the influence of particle resonances on the stability of thin Harris-type current sheets. In our analytical investigations, we avoid the limitation of the slab approximation, usually used in the theory of thick current sheets. Instead we solved the generalized eigenvalue problem algebraically by applying an appropriate system of base functions which smoothly cover the whole spatial domain of interest. This also allowed us to avoid the $A_{1}(Z) \approx A_{1}(0)=$ const. approximation, usually used in the thick sheet tearing mode theory to describe the resonance interaction in a narrow slab near the center of the sheet. The eigenfunctions $A_{1}^{(n)}(Z)$ also allowed us a determination of the symmetry properties of the fastest growing unstable waves. We conclude that the threshold of instability is reached as soon as the sheet thickness becomes comparable with the thermal ion Larmor radius in the external magnetic field, i.e., after the sheet has thinned down to the ion inertial length. This agrees with the prediction of Yamanaka (1987). We further found that, after the current sheet half-thickness reaches the threshold a bulk current instability of the whole sheet starts to grow. The fastest growing mode is symmetric about the neutral plane. We called it a kinetic sausage mode since it reminds the similar ideal MHD instability. The resulting sausage mode wave propagates in the current direction. Only for artificially small values $M=M_{i} / m_{e}=\mu^{-1}<10$ growth rate and wave length strongly depend (due to the influence of the lighter particles, the "electrons") on the mass ratio $M$. For larger mass ratios the damping electron influence disappears. The theoretically predicted wave number of maximum growth is $L_{z} k \approx 2$. The symmetry of our analytical solution of the non-local linear kinetic dispersion relation agrees with the numerically obtained ones of Lapenta and Brackbill (1997) and contradicts their theoretical results. Our investigations cannot be directly compared with those of Pritchett et al. (1996), since they solved an initial value problem and did not look for the natural eigenmodes of the system. In the long wavelength limit our theoretical results agree with those reached by Yoon et al. (1998) in a two-fluid approach, but not for shorter wavelengths. This is due to the neglect of resonance effects in the twofluid approach, which damp the shorter wavelength mode growth. 
In the past, our kinetic PIC code plasma simulations had already revealed a sausage mode in the mass ratio $M=1$ limit and for relatively short boxes in the current direction (Büchner and Kuska, 1996, 1997). In order to verify these results and to compare with the linear stability analysis, we have repeated these simulations varying the box length in the current direction, the number of particles, the temperatures, the spatial (Debye length) resolution, the aspect ratio of the simulation box and the size of the embedded current sheet. We started with marginally thin $\left(L_{z}=\rho_{0, i}\right)$ current sheets in Harris equilibria. We did not include any background plasma as it was done in other codes by technical reasons. In this paper we have shown representative results for mass ratios $M=1$ and $M=64$. In contrast to the simulations of Zhu and Winglee (1996), Ozaki et al. (1996), Pritchett et al. (1996), Lapenta and Brackbill (1997), our simulations confirm the prevalence of the sausage mode bulk current instability over the asymmetric kink mode. A comparison of the simulation results with the theoretically obtained properties of the bulk current instability shows that they correspond well with the theoretical results.

We can only guess, at the moment, the reason for the asymmetry of the (kink) mode in the other simulations. Different reasons are thinkable, which may break the symmetry and lead to sheet kinking instead of the sausage symmetry. One group of possible reasons is semi-physical. In the presence of a resting background plasma a Kelvin-Helmholtz instability may grow at the interface between the fast-flowing, current-carrying particles at the edge of the current sheet. Indeed, many simulation codes use a plasma background to avoid numerical instabilities. Since the particle drifts are often assumed to be artifically high (by technical reasons) the threshold of a Kelvin Helmholtz instability can be reached even for perturbations with wave vectors perpendicular to the magnetic field direction. Another group of reasons for symmetry breaking could be due to technical asymmetries inherent, e.g., to the usually used leapfrog methods. They take field values calculated during different time steps when determining derivatives. Other reasons might by effects of the high particle velocities due to incorrect transformations of the superrelativistic speeds due to plasma heating. The GISMO code was intentionally developed to avoid these problems. All symmetry breaking effects thinkable should be checked in order to verify the mode of the instability of thin current sheets properly.

In separate papers we have shown that the non-local bulk instability of thin current sheets accelerates three dimensional spontaneous reconnection (Büchner and Kuska, 1996, 1997). After verifying the sausage mode instability both theoretically and by means of PIC-code kinetic simulations, we can draw from these results the following conclusions for the onset of reconnection in the course of magnetospheric substorms. First, external pressure and energy inflow cause a current sheet thinning in the near-Earth magnetotail. As soon as the threshold sheet-width comparable with the ion inertial length or half the ion gyroradius is reached, a bulk current instability starts. It heats the electrons above their threshold of non-adiabaticity. This releases the frozen-flux constraint for the electrons and allows a loss of the current sheet equilibrium. Since the growth rate of the instability, on the other hand, is controlled by the ions, the ion scale determines the rate at which reconnection evolves (Büchner, 1998). Extrapolating the kinetic plasma simulation results to realistic mass ratios by using the predictions of the linear dispersion relation, one obtains that the characteristic growth time of the sausage-mode bulk current instability of marginally thin sheets would be of the order of a minute. This agrees well with the observed time-scale of substorm onsets.

Acknowledgement. Topical Editor K.-H. Glassmeier thanks, M. Scholer and another referee for their help in evaluating this paper.

\section{References}

Axford, I., Magnetic field reconnection, in Reconnection in space and Laboratory Plasma, ed. E. W. Hones Jr., Geophysical Monograph 30, AGU, Washington D.C., pp. 4-14, 1984.

Baker, D. N., T. I. Pulkkinen, V. Angeleopoulus, W. Baumjohann, and R. L. McPherron, Neutral line model of substorms: past results and present view, J. Geophys. Res., 101, 12,975, 1996.

Baker, D. N., T. I. Pulkkinen, J. Büchner, and A. Klimas, Substorms: a global magnetospheric instability, in Proceedings of the IVth International Conference on Substorms, eds. S. Kokubun and Y. Kamide, Kluwer Dordrecht, 231, 1998.

Birdsall C. K., and A. B. Langdon, Plasma Physics via Computer Simulation, Plasma Physics Series, IOP Publishing, 1991.

Brackbill, J. U., D. W. Forslund, K. B. Quest, and D. Winske, Nonlinear evolution of the lower-hybrid drift instability, Phys. Fluids, 27, 2682, 1984.

Büchner, J., Multiscale coupling in reconnection - three-dimensional current sheet tearing, in Physics of Space Plasmas, eds. T. Chang, G. B. Crew and J. R. Jasperse, MIT Cambridge, MA, pp. 79, 1995.

Büchner, J., Kinetic effects controlling the onset of $3 \mathrm{D}$ reconnection in Proceedings of the IVth International Conference on Substorms, eds. S. Kokubun and Y. Kamide, Kluwer, Dordrecht, 461, 1998.

Büchner, J., and J.-P. Kuska, Three-dimensional collisionless reconnection through thin current sheets: theory and selfconsistent simulations, in Proceedings of the IIIrd International Conference on Substorms, ESA SP-389, October 1996, 373, 1996.

Büchner, J., and J.-P. Kuska, Numerical simulation of threedimensional reconnection due to the instability of collisionless current sheets, Adv. Space Res., 19, 1817, 1997.

Büchner, J., and, L. M. Zelenyi, Chaotization of the electron motion as the cause of an internal magnetotail instability and substorm onset, J. Geophys. Res., 92, 13,456, 1987.

Büchner, J., and L. M. Zelenyi, Regular and chaotic charged particle motion in magnetotail-like field reversals. 1 Basic theory, J. Geophys. Res., 94, 11,821, 1989.

Cash, J. R., and, A. H. Karp, A variable order Runge-Kutta method for initial value problems with rapidly varing righthand sides, ACM Transactions on Mathematical Software, 16, 201, 1990.

Coppi, B., G. Laval, and R. Pellat, A model for the influence of the Earth magnetic tail on geomagnetic phenomena, Phys. Rev. Lett., 16, 1207, 1966.

Coroniti, F. V., Space plasma turbulent dissipation: reality or myth?, Space Sci. Rev., 42, 399, 1985.

Coroniti, F. V., and A. Eviatar, Magnetic field line reconnection in a collisionless plasma, Astrophys. J. Suppl. Ser., 33, 189, 1977. 
Deufihard, P., and F. Bornemann, Numerische Mathematik II: Integration gewöhnlicher Differentialgleichungen, de Gruyter, Berlin, 1994.

Hairer, E., S. P., Norsett, and G. Wanner, Solving ordinary differential equations. I. Nonstiff problems, Springer Series in Computational Mathematics 8, Springer, Berlin Heidelberg, New York 1987.

Harris, E. G., On a plasma sheath separating regions of oppositely directed magnetic field, Nuovo Cimento, 23, 115, 1962.

Hones, E. W. Jr., Plasma flow in the magnetotail and its implications for substorms theories, in Dynamics of the Magnetosphere, ed. S.I. Akasofu, 545, D. Reidel, Dordrecht, 1979.

Huba, J. D., N. T. Gladd, and K. Papadopoulus, The lower hybrid drift instability as a source of anomalous resisitivity for magnetic reconnection, Geophys. Res. Lett., 4, 125, 1977.

Huba, J. D., J. F. Drake, and N. T. Gladd, Lower hybrid drift instability in field reversed plasmas. Phys. Fluids, 23, 552, 1980.

Kaufmann, L. R., Substorm currents: growth phase and onset, J. Geophys. Res., 92, 7471, 1987.

Kuznetsova, M. M., and B. Nikutowski, A modified lower hybriddrift instability as a possible mechanism for the generation of magnetic noise bursts in the magnetotail neutral sheet, J. Geophys. Res., 99, 4105, 1994.

Lapenta, G., and J. U. Brackbill, A kinetic theory for the drift-kink instability, J. Geophys. Res., 102, 27,099, 1997.

Lui, A. T. Y., C.-L. Chang, A. Mankofsky, H. K. Wong, and D. Winske, A cross-field current instability for substorm expansion, J. Geophys. Res., 96, 11,389, 1991.

Mitchell, D. G., Current carriers in the near-Earth's cross-tail current sheet during a substorm growth phase, Geophys. Res. Lett., 17, 583, 1990.

Ozaki, M., T. Sato, R. Horiuchi, and C. S. Group, Electromagnetic instability and anomalous resistivity in a magnetic neutral sheet, Phys. Plasmas, 3, 2265, 1996.

Pellat, R., F. Coroniti, and P. Pritchett, Does the ion tearing exist? Geophys. Res. Lett., 18, 143, 1991.

Pritchett, P. L., Effect of electron dynamics on collisionless reconnection in two-dimensional magnetotail equilibria, J. Geophys. Res., 99, 9935, 1994.

Pritchett, P. L., and J. Büchner, Collisionless reconnection in configurations with a minimum in the equatorial magnetic field and with magnetic shear, J. Geophys. Res., 100, 3601, 1995.

Pritchett, P. L., and F. V. Coroniti, The role of the drift kink mode in destabilizing thin current sheets, J. Geomag. Geoelectr., 48, 833,1996
Pritchett, P. L., F. V. Coroniti and V. K. Decyk, Three-dimensional stability of thin quasi-neutral current sheets, J. Geophys. Res., 101, 27,413, 1996.

Pulkkinen, T., D. N. Baker, R, J. Pellinen, J. Büchner, H. E. J. Koskinen, R. E. Lopez, R. L. Dyson, and L. A. Frank, Particle scattering and current sheet stability in the geomagnetic tail during the substorm growth phase, J. Geophys. Res., 97, 19,283, 1992.

Russell, C. T., and R. L. McPherron, The magnetotail and substorms, Space Sci. Rev., 15, 205, 1973.

Sanny, J., R. L. McPherron, C. T. Russell, D. N. Baker, T. I. Pulkkinen, and A. Nishida, Growth-phase thinning of the nearEarth current sheet during the CDAW 6 substorm, J. Geophys. Res., 99, 5805, 1994.

Schindler, K., A theory of substorm mechanisms, J. Geophys. Res., 79, 2803, 1974.

Schindler, K., and J. Birn, On the cause of thin current sheets in the near-Earth magnetotail and their possible significance for magnetospheric substorms. J. Geophys. Res., 98, 15,477, 1993.

Sergeev, V., P. Tanskanen, K. Mursula, A. Korth, and R. C. Elphic, Current sheet thickness in the near-Earth plasma sheet during substrom growth phase, J. Geophys. Res., 95, 3819, 1990.

Sonnerup, B. U., Adiabatic particle orbits in a magnetic null sheet, J. Geophys. Res., 76, 8211, 1971.

Speiser, T., Particle trajectories in model current sheets, J. Geophys. Res., 70, 4219, 1965.

Vasyliunas, V. M., Theoretical models of magnetic field linemerging, 1, Rev. Geophys. Space. Phys., 13, 303, 1975.

Verner, J. H., Differentiable interpolants for high-order RungeKutta methods, SIAM J. Numer. Anal., 30, 1446, 1993.

Wiegelmann, T., and K. Schindler, Formation of thin current sheets in a quasistatic magnetotail model, Geophys. Res. Lett. 22, 2057, 1995.

Winske, D., Current-driven microinstability in a neutral sheet, Phys. of Fluids, 24, 1069, 1981 a.

Winske, D., Particle simulation studies of the lower hybrid drift instability, Phys. Fluids, 24, 1069, 1981 b.

Yamanaka, K., Threshold of electromagnetic instability in a magnetic neutral sheet, Physica Scripta, 17, 15, 1978.

Yoon, P. H., A. T. Y. Lui, and H. K. Wong, Two-fluid theory of drift-kink instability in a one-dimensional neutral sheet, J. Geophys. Res., 103, 11,875, 1998.

Zhu, Z., and R. M. Winglee, Tearing instability, flux ropes, and the kinetic current sheet kink instability, J. Geophys. Res., 101, $4885,1996$. 